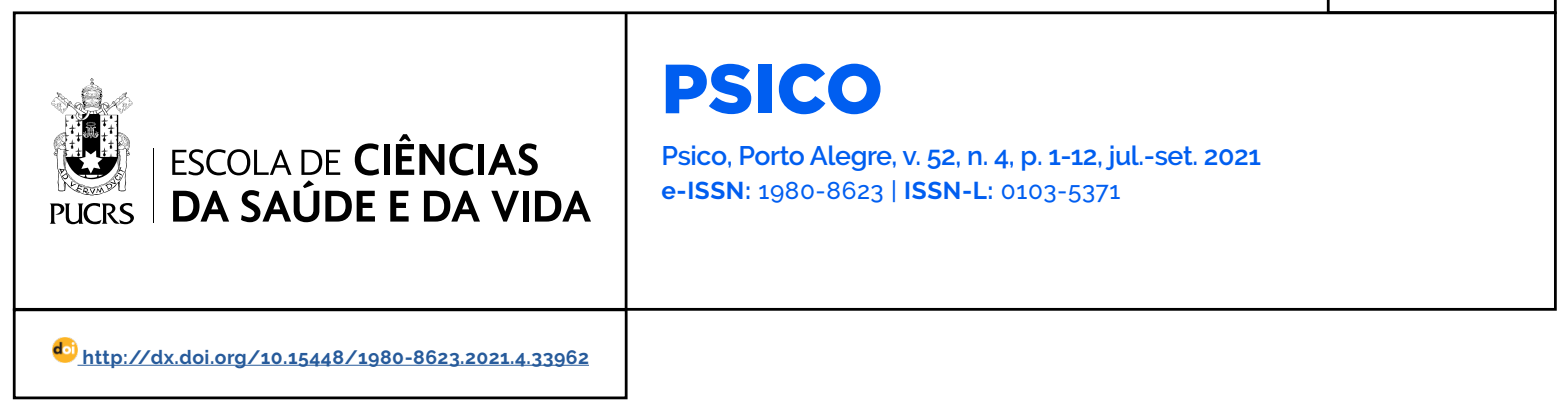

SEÇÃO: ARTIGO

\title{
Avaliação de intervenção para promoção de resiliência em familias de crianças com Síndrome de Down
}

\author{
Assessment of intervention to promote resilience in families of children with Down Syndrome \\ Evaluación de intervención para promoción de resiliencia en familias de niños con \\ Sindrome de Down
}

\section{Mayse Itagiba Rooke ${ }^{1}$}

orcid.org/0000-0002-4510-4327

mirpsi@gmail.com

\section{Nara Liana Pereira}

Silva ${ }^{1}$

orcid.org/0000-0002-8435-3078

naralianaps@gmail.com

Recebido em: 20 abr. 2019.

Aprovado em: 14 set. 2020

Publicado em: 19 jan. 2022.
Resumo: Este estudo objetiva avaliar efeitos de uma intervenção que busca promover resiliência em oito familias de crianças com sindrome de Down (SD) de até dois anos de idade. Classificou-se as familias segundo arranjos e renda, distribuídas aleatoriamente nos grupos experimental e controle. Visitas domiciliares ocorreram no pré-teste, pós-teste e follow up, onde todos responderam ao questionário sociodemográfico e à entrevista acerca da resiliência familiar, além dos genitores responderem ao Inventário de Sintomas de Estresse de Lipp e ao Inventário de Depressão de Beck. Um diário de campo e entrevistas de avaliação para as familias foram realizados durante a intervenção. Não foram obtidas diferenças estatisticamente significativas entre os valores da resiliência nas familias nos diferentes momentos $(p=0,77)$. Entretanto, alguns membros percebem mudanças na familia após participação no trabalho, como melhorias na comunicação e aquisição de conhecimentos sobre SD. Estudos com mais participantes e periodo de tempo prolongado são necessários.

Palavras-chave: resiliência familiar, intervenção, familia, sindrome de down

Abstract: This study aims to evaluate effects of an intervention to promote resilience in eight families of children with Down syndrome (DS) up to 2 years of age. These families were classified according to arrangements and income, then, they were randomly distributed into experimental and control groups. Home visits occurred in three steps: pre-test, post-test and follow up. All participants answered a sociodemographic questionnaire and a family resilience interview: in addition, parents responded to Lipp Stress Inventory and Beck Depression Inventory. A field diary and an evaluation interview for the families were conducted during intervention. No statistically significant differences were found between family resilience values at different steps $(p=0,77)$. However, some participants perceive changes in their family after intervention, for example, improvements in communication and knowledge about DS. Studies with more participants and extended period of time are needed.

Keywords: family resilience, intervention, family, down syndrome

Resumen: Este estudio objetivó evaluar los efectos de una intervención que busca promover la resiliencia en ocho familias de niños con sindrome de Down (SD) de hasta 2 años de edad. Las familias fueron clasificadas según su tipologia y sus ingresos, distribuidas aleatoriamente en los grupos experimental y control. Las visitas domiciliarias fueron realizadas en tres momentos, donde todos respondieron a un cuestionario y a una entrevista sobre resiliencia familiar, además los progenitores respondieron a inventarios de estrés y depresión y entrevistas de evaluación de la intervención. Se utilizó un diario de campo. No se obtuvo diferencias estadisticamente significativas entre los valores de la

\section{(c) (1)}

Artigo está licenciado sob forma de uma licença Creative Commons Atribuição 4.0 Internacional. 
resiliencia en las familias en los momentos $(p=0,77)$. Sin embargo, algunos miembros percibieron cambios después de participar en el estudio, como mejoras en la comunicación y adquisición de conocimientos sobre el SD. Son necesarias investigaciones con más participantes y un periodo de tiempo prolongado.

Palabras clave: resiliencia familiar, intervención, familia, sindrome de down

A sindrome de Down (SD) é causada pela presença de três cromossomos $21 \mathrm{em}$ todas ou na maior parte das células de um indivíduo, sendo uma condição genética que traz implicações em vários aspectos do desenvolvimento e do organismo da pessoa. As crianças com essa sindrome apresentam deficiência intelectual, hipotonia muscular e atraso na aquisição de marcos motores (Goff et al., 2016; Marchal et al., 2017; Nussbaum et al. 2015). Paralelamente a estas caracteristicas, pessoas com esse diagnóstico apresentam, frequentemente, predisposição para comorbidades patogênicas, tais como cardiopatia congênita, doença gastrointestinal, hipotireoidismo e problemas respiratórios (Aburawi et al., 2015: Roizen et al., 2014). Em consequência dessas características e desses problemas de saúde, a criança com SD demanda mais cuidado do que aquela com desenvolvimento típico (DT), podendo gerar sobrecarga, altos niveis de estresse e depressão nos genitores (Abbasi et al., 2016; Alexander \& Walendzik, 2016; Goff et al., 2016). É consenso na literatura que níveis elevados de estresse e depressão dos genitores influenciam negativamente as relações de parentalidade, promovendo problemas no funcionamento familiar, o que pode dificultar o desenvolvimento de resiliência nessas familias (Gerstein et al., 2009; Grant et al., 2013). Além desses aspectos, pais e mães ainda têm de lidar com preocupações referentes à aprendizagem, à aceitação social, ao sucesso e à autonomia do filho com deficiência (Azevedo \& Damke, 2017; Choi \& Yoo, 2015).

Nesse sentido, não há dúvidas que as implicações da SD tendem a colocar o grupo familiar em situações de vulnerabilidade. Contudo, a literatura identifica alguns genitores como capazes de gerenciar os impactos da deficiência em suas vidas. Por exemplo, Scorgie e Sobsey (2000) e Van Riper (2007) evidenciaram em seus estudos o desenvolvimento de propósito de vida e de compaixão e fortalecimento de crenças espirituais. Crescimento pessoal e rearranjo de prioridades (Choi \& Van Riper, 2016), além de estreitamento de vinculos intrafamiliares e organização de forma cooperativa com diálogos diante de problemas (Rooke \& Pereira-Silva, 2016) também foram caracteristicas positivas presentes em familias com filhos com SD.

Para que familias consigam se fortalecer/ empoderar, a literatura aponta que é importante a promoção de recursos extrafamiliares por meio de, por exemplo, realização de programas de intervenção dirigidos a elas (Choi, Lee, \& Yoo, 2011; Crnic et al., 2017; Koros \& Harrahs, 2017). Nesse sentido, Choi et al. (2011) destacam que o momento de receber a notícia do diagnóstico da SD é vivenciado com preocupação e insegurança, salientando que os genitores que participaram de uma intervenção em grupo com objetivo de oferecer suporte emocional e informações referentes à sindrome, relataram mais respostas emocionais positivas ao enfrentarem os desafios advindos da parentalidade de um filho com SD do que os genitores que não participaram. Destaca-se, portanto, a importância de serviços voltados a essas pessoas, o que poderá promover processos de resiliência familiar, permitindo ao grupo superar crises e enfrentar dificuldades prolongadas (Koros \& Harrahs, 2017; Padesky \& Mooney, 2012; Walsh, 2016).

De acordo com Walsh (2016), a resiliência em familias consiste no interjogo de três processos-chave: (a) sistemas de crenças, (b) padrões de organização e (c) processos de comunicação. O sistema de crenças é definido como os valores, convicções e suposições que se misturam para desencadear um conjunto de reações emocionais, decisões e ações. Os padrões de organização referem-se ao modo das familias mobilizarem recursos, de resistirem ao estresse e de se reorganizarem para se adequar às condições modificadas. Os processos de comunicação consistem nas formas como são realizadas as trocas de informações entre pessoas. Em uma família com indicativos de resiliência, caracterizam- se 
por serem diretos, nos quais os membros familiares são capazes de demonstrar e de tolerar um amplo repertório de sentimentos, bem como resolver problemas de forma cooperativa.

No que se refere às intervenções que objetivam promover a resiliência, Padesky e Mooney (2012) salientam que podem ajudar as familias a identificar seus pontos fortes, reconhecer os fatores protetores, recursos internos e do ambiente que podem ser utilizados e desenvolvidos, além de fornecer estratégias especificas para melhorar o funcionamento e o enfrentamento positivo da familia frente a uma adversidade. Embora possam se constituir uma valiosa forma de nortear esforços no campo de prevenção e de promoção de saúde, essas intervenções ainda são escassas (Oñate \& Calvete, 2017; Sánchez-Teruel $\&$ Robles-Bello, 2015), especialmente aquelas que são avaliadas no que tange aos seus efeitos (Dempsey \& Keen, 2008). Por conseguinte, este trabalho tem como objetivo avaliar os efeitos de uma intervenção com foco na promoção de resiliência em famílias de crianças com SD de até dois anos de idade.

\section{Método}

Este tópico descreve os aspectos metodológicos que foram adotados neste trabalho, destacando-se os participantes, os instrumentos que foram utilizados, os procedimentos para coleta e análise de dados, além das considerações éticas. Ressalta-se que se trata de um estudo com delineamento quase experimental e longitudinal, já que: (a) o estudo buscou estabelecer uma relação de causa-efeito, na qual foi investigado se a intervenção desenvolvida e implementada nas familias pertencentes ao grupo experimental (GE) propiciou processos de resiliência familiar; e (b) o estudo envolveu a manipulação da variável independente, a intervenção, em diferentes grupos, GE e controle (GC) ao longo do tempo, possibilitando a observação de efeitos dessa manipulação na variável dependente, a resiliência familiar (Santos, 2007).

\section{Participantes}

Participaram do estudo oito famílias e, no que tange aos seus arranjos, seis foram classificadas como nucleares tradicionais e duas, como recasadas. Destaca-se que em quatro familias, além do filho com SD, existia também filhos com DT. A idade média das mães foi de 37.9 anos (DP = 7.3: $\mathrm{Md}=37,5)$, enquanto dos pais, de 39,5 anos $(\mathrm{DP}=5,5 ; \mathrm{Md}=38,5)$, das crianças com SD, de 15,1 meses (DP $=7,2 ; \mathrm{Md}=16$ ) e dos filhos com DT, de 10,6 anos (DP = 5,3; Md = 4). Seis mães eram do lar e duas apresentavam vínculos empregatícios, enquanto todos os pais trabalhavam fora de casa. A renda familiar foi, em média, de 6,2 salários-mínimos (DP = 4,5; $\mathrm{Md}=4,2$ ).

\section{Instrumentos}

Questionário sociodemográfico. Um breve questionário foi construído para identificar dados demográficos da família, como idade dos membros, profissão e renda.

Inventário de sintomas de estresse para adultos de Lipp (ISSL - Lipp, 2000). O ISSL é composto por três quadros com 37 itens fechados referentes à sintomatologia somática e 19 relacionados à psicológica, sendo os sintomas, geralmente, repetidos, diferindo somente em sua intensidade e seriedade. Este instrumento tem o objetivo de medir a frequência de sintomas de estresse, o tipo de sintoma existente (se somático ou psicológico) e a fase em que o respondente se encontra (Alerta, Resistência, Quase-Exaustão e Exaustão). Foram realizadas análises de consistência e de confiabilidade do instrumento (alfa de Cronba$c h=0,9121$ ), análise fatorial e agrupamento dos itens, ambas com resultados que demonstram que o ISSL pode ser considerado como um bom e válido instrumento de avaliação do estresse.

Inventário de depressão de Beck (BDI-II- Beck et al., 1996). O BDI consiste em uma escala de autopreenchimento com 21 grupos de afirmações sobre os sintomas depressivos. Cada questão do inventário possui pontuações crescentes dispostas em uma escala tipo Likert, cujos escores variam de o a 3. Este inventário objetiva detectar 
sintomas depressivos em individuos a partir de 13 anos. Gorenstein, Wang et al. (2011) realizaram a adaptação brasileira do inventário, sendo a confiabilidade do BDI-II avaliada por intermédio de diversos indicadores psicométricos de fidedignidade ou precisão, dentre eles o coeficiente de consistência interna alfa de Cronbach com valor em torno de 0,90.

Roteiro para entrevista semiestruturada acerca da resiliencia familiar. Este instrumento é formado por 11 questões, tendo sido construido a partir da literatura acerca da resiliência familiar de Walsh (2016) e utilizado em Rooke (2014, 2019). O roteiro objetivou identificar o estágio do processo de resiliência nas familias a partir da análise dos três processos-chave.

Roteiro para entrevista semiestruturada acerca da avaliação da intervenção. O roteiro com sete perguntas abertas foi elaborado pelas próprias pesquisadoras, tendo como objetivo realizar uma avaliação de processo da intervenção por parte de cada membro familiar.

Roteiro para diário de campo. O roteiro com cinco questões abertas foi construido para a realização desta pesquisa. Tal instrumento objetivou descrever como foi a implementação da intervenção no que tange à motivação das famílias em participar dos encontros, aos momentos de dificuldades das pesquisadoras em realizar as oficinas e aos possiveis aprimoramentos necessários no decorrer das atividades para que fosse realizada uma avaliação de processo da intervenção.

\section{Procedimentos}

\section{Coleta de dados}

Posteriormente à aprovação deste estudo pelo Comitê de Ética em Pesquisa com Seres Humanos da Universidade Federal de Juiz de Fora (UFJF), sob CAAE 69341917.4.0000.5147, realizou-se o recrutamento de familias por conveniência. Para tanto, foi feito contato com instituição pública referência na cidade de Juiz de Fora, MG, e redondezas, que oferece serviços de atendimento para crianças com SD. Assim, prontuários foram consultados a fim de selecionar crianças diagnosticadas com SD com idade entre um mês e dois anos. No total, 17 familias foram identificadas e contatadas por telefone e todas aquelas que apresentaram disponibilidade para colaborar com o estudo foram inclusas na amostra. Ademais, cinco familias optaram por não participar da pesquisa, alegando, principalmente, a ausência de tempo para receber as pesquisadoras em suas residências. Destaca-se que duas outras familias aceitaram colaborar com o estudo, no entanto, não foram selecionadas por estarem em processo de mudança para outro município.

A partir do recrutamento, foi marcada uma primeira visita à residência das famílias $(n=10)$ em dias e horários disponibilizados por elas, iniciando o primeiro momento do estudo, denominado de pré-teste e realizado com todas as familias. Foram necessárias duas visitas domiciliares para que: (a) o consentimento livre e esclarecido do pai, da mãe e do filho com DT fossem obtidos, (b) o questionário sociodemográfico fosse aplicado de forma conjunta em todos os membros familiares, (c) os instrumentos BDI e ISSL fossem respondidos, separadamente, pelos genitores, tendo sido aplicados pelas pesquisadoras e (d) a entrevista sobre os aspectos que envolvem os processos-chave de resiliência familiar fossem gravadas em áudio com todos os membros familiares separadamente. O tempo médio de duração para cada visita familiar foi de 90 minutos.

Após essas duas visitas, as familias foram classificadas de acordo com tipo de arranjo familiar (nuclear tradicional e recasada) e renda familiar, sendo, posteriormente, distribuidas aleatoriamente em dois grupos, GE e GC, cada grupo com cinco famílias. A decisão de classificar e dividir as famílias em grupos de acordo com tais caracteristicas (arranjo e renda familiar) foi realizada, uma vez que: (a) relaciona-se com resultados de estudos que mostram que, em geral, pais e mães solteiros, geralmente, têm menos recursos financeiros, mais sobrecarga de funções, e enfrentam mais desafios na gestão de funções parentais em comparação com genitores casados (Mazzeo, 2007; Raposo et al., 2009), o 
que pode causar problemas de saúde mental e menor percepção de apoio social, que influenciam na forma como o grupo familiar lida com as adversidades e, portanto, na resiliência familiar; e (b) a partir de distribuições similares, os grupos tornam-se mais comparáveis, de forma que as associações estimadas podem ser atribuidas de forma mais concisa à intervenção realizada com o grupo experimental.

A partir das distribuições das familias em dois grupos, GE e GC, deu-se início ao segundo momento desta pesquisa, denominado de implementação da intervenção no GE. Vale ressaltar que no GC os membros familiares apenas responderam aos instrumentos da presente pesquisa, sem participar de qualquer intervenção. Neste momento, em função de imprevistos ocorridos com duas familias, elas desistiram de participar do estudo e, por conseguinte, oito familias permaneceram como participantes deste estudo, quatro no GE e quatro no GC.

A intervenção no GE consistiu em 11 encontros semanais acerca do sistema de crenças da familia, dos padrões de organização familiar e dos processos de comunicação, conforme detalhado em Rooke (2019). Oito oficinas foram realizadas na residência de cada familia separadamente e três tiveram a participação de todas as familias do GE ao mesmo tempo. No total, a intervenção teve duração de, aproximadamente, 13 horas e 21 minutos para cada familia, ao longo de cinco meses. Cada oficina domiciliar teve duração, em média, de 68 minutos, e a oficina em grupo teve duração, em média, de 87 minutos. Ao final de cada encontro era preenchido um diário de campo. Destaca-se que as familias do GC não foram visitadas nem contatadas durante o tempo em que as famílias do GE participavam da intervenção.

Findada a intervenção, logo em seguida e de acordo com a disponibilidade de cada família, aproximadamente no intervalo de sete dias, foi iniciado o terceiro momento da pesquisa, intitulado de pós-teste, com uma visita domiciliar em cada uma das familias do GE e GC com duração de, aproximadamente, 94 minutos cada. Os ge- nitores e filhos com DT, individualmente, foram entrevistados acerca do processo de resiliência familiar e pais e mães responderam ao ISSL e BDI. Nesse momento, as familias do GE também foram entrevistadas, separadamente, em relação à avaliação da intervenção.

Vale ressaltar que o momento do pós-teste com as familias do GE contou com a participação de duas pesquisadoras não habituais treinadas para conduzir o encontro com as familias. Essas mesmas pesquisadoras aplicaram os instrumentos em ordem aleatória. Essas estratégias foram adotadas para controle de desejabilidade social e efeito de ordem.

Após quatro meses da última visita domiciliar em cada família, foi realizado um follow up, dando início ao quarto e último momento desta pesquisa, no qual todas as famílias responderam aos mesmos instrumentos do momento anterior, exceto a entrevista de avaliação da intervenção. A duração média da visita domiciliar nesse momento foi de 97 minutos. Ao final do follow up, as familias do GC foram convidadas a participar da intervenção. Cabe salientar que a pesquisa se iniciou em outubro de 2017, tendo sido finalizada em outubro de 2018, isto é, foi necessário 1 ano para que fosse concluida.

\section{Análise dos dados}

Os instrumentos ISSL e BDI-II foram analisados de acordo com os respectivos manuais para correção. Os dados do questionário sociodemográfico foram tabulados, tendo sido construido um banco com as informações de todas as familias. Posteriormente, por meio do programa SPSS (Statiscal Package for the Social Sciences) para Windows, versão 20.0, efetuou-se cálculos com base em estatística descritiva, especificamente medidas de dispersão, como média, desvio padrão e mediana para caracterizar as familias participantes do estudo.

As entrevistas em relação à avaliação da intervenção foram transcritas na integra e analisadas pelas pesquisadoras, conjuntamente, com base na proposta de Dessen e Cerqueira-Silva (2009), que prevê a construção do Sistema Integrado de Categorias complementar à análise de conteúdo 
proposta por Bardin (2011). Este modelo pressupõe os passos destacados por Bardin: (a) seleção e exploração do material, denominada pré-análise; (b) codificação; (c) agrupamento dos temas; (d) formação das categorias sintese; (e) classificação dos temas; (f) definição das categorias. Complementarmente, Dessen e Cerqueira-Silva (2009) propõem: (g) revisão do sistema preliminar e elaboração do sistema integrado (definitivo) de categorias e (h) validação do sistema integrado de categorias, a partir da análise de juizes.

Já para a análise das entrevistas acerca da resiliência familiar seguiu os seguintes passos: (a) transcrição das entrevistas gravadas em áudio, (b) construção de um instrumento que possibilitasse a identificação de fases da resiliência familiar e (c) preenchimento do instrumento e cálculo da média entre as variáveis de resiliência familiar percebida pela mãe e pelo pai com auxilio do programa SPSS para Windows, versão 20.0, de forma que cada família tivesse um escore para a variável resiliência familiar.

Destaca-se que o instrumento foi construido tendo como base as respostas dos participantes a cada questão, havendo a possibilidade de analisar cada questão e indicar se o relato se aproxima de indicativos do estágio de Latência ou Prosperidade. O estágio de Latência refere-se a relatos que indicam aspectos negativos no que tange aos três processos-chave de Froma Walsh que caracterizam o processo de resiliência (sistema de crenças, padrões de organização e processos de comunicação), resultando na ausência de indicativos de resiliência familiar, porém considerando que a familia tem habilidades para desenvolvê-la. Foram classificados como estando no estágio de Prosperidade aquelas "falas" em que havia presença de aspectos positivos acerca dos processos-chave, apontando indicativos de resiliência familiar, isto é, o grupo familiar adaptou-se positivamente à adversidade e consegue reconhecer crescimento e aprendizagem devido à vivência da situação de adversidade. Por exemplo: (a) controle do próprio individuo e/ou sua família sob o diagnóstico de sindrome de Down da criança, geralmente, pela culpabilização/atribuição de algum membro familiar (o que equivale a um escore no valor de -1) ou (b) culpabilização/atribuição do divino e/ ou alguma força superior pelo diagnóstico de sindrome de Down na criança, porém como uma oportunidade de aprendizagem e crescimento pessoal e/ou como uma forma de confiança de que o individuo e sua familia têm capacidade para enfrentar positivamente (o que equivale a um escore no valor de +1).

Por meio do programa SPSS para Windows, versão 20.0, também foram calculadas as médias de depressão e estresse para analisar os efeitos da intervenção. Estatísticas não paramétricas, em especial que utilizam o sistema de postos foram favorecidas por não possuírem pressupostos como normalidade e homogeneidade de variância dos dados (Field, 2009). Nesse sentido, para identificar se existia diferença entre os diferentes momentos de aplicação dos instrumentos, foram realizadas comparações de postos de cada processo-chave da resiliência familiar. Para tal, foi utilizado o teste

ANOVA de Friedman, que visa identificar diferença entre mais de dois grupos.

O teste Wilcoxon foi utilizado para comparar se os grupos experimental e controle diferem em média em todas as variáveis avaliadas. O teste Wilcoxon é um teste não paramétrico para comparação de grupos, recomendado quando não há indicativo de normalidade nos dados. Neste teste, é esperado que o valor de significância ( $p$ ) seja menor que 0,05 e que o intervalo de confiança não ultrapasse o eixo o para que se considere que os grupos possuem valores diferentes.

\section{Resultados}

No que tange à avaliação de impacto da intervenção, o teste ANOVA de Friedman não identificou diferença significativa entre os valores da média de resiliência familiar $(p=0,77)$ nem dos três processos-chave, sistemas de crenças, padrões de organização e processos de comunicação ( $p=0,30, p=0,18, p=0,58$, respectivamente) que a caracterizam nos diferentes tempos de mensuração. 
Já o teste de comparação intergrupos demonstrou que os grupos experimental e controle foram diferentes para as médias das variáveis "Resiliência familiar/Dimensão 1" no pós-teste (I.C. $95 \%=-3,50-0,50, p<0,05)$, "Depressão" (I.C.
$95 \%=7.5023 .50, p<0,05)$ e "Estresse" (I.C. $95 \%=$ $5,50$ 26,00, $p<0,05)$, sendo estas no pré-teste, conforme pode ser observado na Tabela 1.

Tabela 1 - Comparação Intergrupo, Segundo Teste Wilcoxon

Intervalo de confiança

\begin{tabular}{|c|c|c|c|c|}
\hline & valor $W$ & $\begin{array}{c}\text { valor } \\
p\end{array}$ & $\begin{array}{l}\text { Limite } \\
\text { inferior }\end{array}$ & $\begin{array}{l}\text { Limite } \\
\text { superior }\end{array}$ \\
\hline Resiliência familiar no pré-teste & 6,50 & 0,77 & $-10,00$ & 10,00 \\
\hline Resiliência familiar/Dimensão 1 no pré-teste & 6.50 & 0,77 & $-4,00$ & 3,00 \\
\hline Resiliência familiar/Dimensão 2 no pré-teste & 7,50 & 1,00 & $-3,00$ & 4.50 \\
\hline Resiliência familiar/Dimensão 3 no pré-teste & 6,00 & 0,66 & -4.50 & 2,50 \\
\hline Resiliência familiar no pós-teste & 4,00 & 0,60 & $-5,00$ & 3.50 \\
\hline Resiliência familiar/Dimensão 1 no pós-teste* & 0,00 & 0,05 & -3.50 & $-0,50$ \\
\hline Resiliência familiar/Dimensão 2 no pós-teste & 5,50 & 1,00 & $-1,50$ & 3,00 \\
\hline Resiliência familiar/Dimensão 3 no pós-teste & 7,00 & 0,85 & $-2,50$ & 4,00 \\
\hline Resiliência familiar no follow up & 5.50 & 1,00 & $-6,00$ & 7,00 \\
\hline Resiliência familiar/Dimensão 1 no follow up & 8,50 & 0,46 & $-0,50$ & 3,00 \\
\hline Resiliência familiar/Dimensão 2 no follow up & 5.50 & 1,00 & $-3,00$ & 2,00 \\
\hline Resiliência familiar/Dimensão 3 no follow up & 4,00 & 0,60 & $-2,50$ & 2,50 \\
\hline Depressão no pré-teste* & 16,00 & 0,03 & 7.50 & 23.50 \\
\hline Depressão no pós-teste & 14,00 & 0,11 & -5.50 & 22,50 \\
\hline Depressão no follow up & 10,50 & 0,15 & -7.50 & 24,00 \\
\hline Estresse no pré-teste* & 16,00 & 0,03 & 5.50 & 26,00 \\
\hline Estresse no pós-teste & 12,50 & 0,25 & $-9,00$ & 21,50 \\
\hline Estresse no follow up & 9,00 & 0,38 & $-4,50$ & 26,00 \\
\hline
\end{tabular}

Nota: O valor de todas as variáveis foi calculado a partir da média entre os escores de pais e mães, exceto em 'Fatores protetores' e suas dimensões, em que existe apenas um escore para cada família. " " refere-se àquelas variáveis que foram diferentes entre os grupos.

Em relação à avaliação de processo da intervenção, todos os membros familiares $(n=10)$ relataram que estão satisfeitos com a forma na qual as atividades foram realizadas. Para identificação dos relatos dos membros familiares, utilizou-se a letra $\mathrm{P}$ para pais, a letra M para mães e as letras FhDT para os filhos com DT quando existentes, seguidas pelas siglas correspondentes ao grupo experimental (GE). Assim, por exemplo, PGE é o pai de uma familia no grupo experimental, MGE é uma mãe no grupo experimental. Seguem alguns relatos: PGE mencionou "Eu achei muito boa a intervenção (...)" e FhDTGE relatou "Eu achei legal." No que se refere aos efeitos da intervenção, 
a maioria dos membros familiares percebe, pelo menos, uma mudança na familia em decorrência do trabalho realizado $(n=8)$, sendo mais frequente a "Melhora na comunicação" ( $n=5)$ e a "Aquisição de conhecimentos sobre SD" $(n=5)$. Um pai e uma mãe de uma mesma familia não reconhecem efeitos decorrentes da intervenção em suas vidas. Todos os efeitos da intervenção percebidos pelas familias podem ser visualizados na Figura 1.

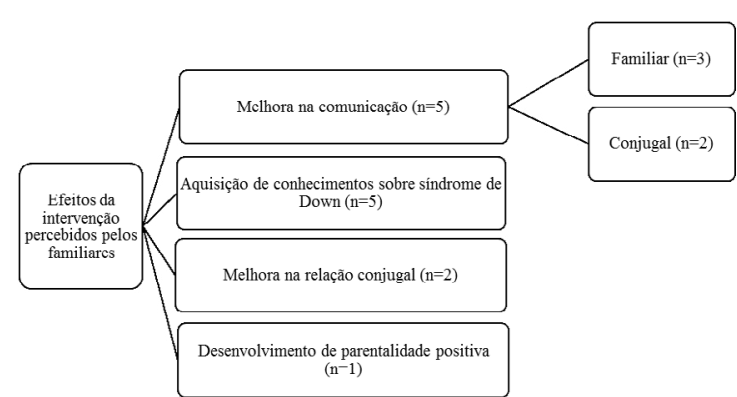

Figura 1 - Efeitos da intervenção percebidos pelas familias.

Abaixo se encontram exemplos de relatos de acordo com as categorias descritas:

(a) Melhora na comunicação:

- Familiar: "(...) ela veio aqui em casa, conversou com a gente, ai fez minha mãe parar um pouco com a gritaria dela, com as brigas que a gente faz." (FhDTGE).

- Conjugal: "Um pouco na comunicação. Agora o N. [referindo-se ao esposo] procura me entender mais também, entender o meu lado." (MGE).

(b) Aquisição de conhecimentos sobre sindrome de Down: "(...) algumas informações que ela [referindo-se à pesquisadora] trouxe a gente até já tinha, outras não (...)" (PGE).

(c) Melhora na relação conjugal: "Eu achei muito boa a intervenção. No nosso caso, foi o que realmente salvou nosso relacionamento, porque a gente não tinha um direcionamento, a gente não sabia para onde que ia mais, não sabia mais o que tinha que fazer." (PGE).

(d) Desenvolvimento de parentalidade positiva: "(...) Colocou a... Pôs a autoestima da gente mais para cima, porque a gente estava pensando, né?
Ficava pensando, né? Por ter ela, né? Com... Especial... Com sindrome de Down. A gente ficava pensando que a gente não ia ser capaz de está conseguindo, é... Conseguir cuidar dela, né?" (MGE).

\section{Discussão}

Como avaliação de impacto dos efeitos da intervenção, pode-se relatar que o teste Anova de Friedman não foi capaz de identificar diferenças na resiliência familiar entre os momentos desta pesquisa. Assim, esse fenômeno psicológico se manteve estável durante os momentos de testagem da pesquisa, isto é, o mesmo resultado do pré-tese permaneceu no pós-teste e no follow up. Ademais, por meio da realização do Teste Wilcoxon, foram detectadas diferenças entre os grupos experimental e controle no que tange ao sistema de crenças da resiliência familiar no pós-teste. Esse resultado favorece interpretações de que o programa de intervenção possa ter tido efeitos em tal variável. No entanto, o processo-chave em questão tende a ser considerado o mais complexo de ser modificado em relação aos padrões de organização e aos processos de comunicação, já que de acordo com Walsh (2016), o primeiro é socialmente construído sob forte influência da família e encontra-se no âmago de quem somos. Ainda segundo a autora, as crenças e as ações, como se organizar e se comunicar são interdependentes. Isto é, as crenças podem facilitar ou restringir as ações, da mesma forma que essas e suas consequências podem reforçar ou alterar as crenças e, portanto, alterações em um processo-chave tendem a modificar outros. Sob esta perspectiva, acredita-se que este resultado deva ser analisado com cautela.

A partir disso, acredita-se que tão importante quanto identificar os efeitos do programa de intervenção, é reconhecer que o periodo de tempo para a realização da presente pesquisa talvez tenha sido pequeno e/ou não suficiente para detectar transições na vida familiar, que tendem a representar impulsos para mudanças no desenvolvimento que, por sua vez, afetam os processos familiares (Dessen \& Braz, 2005) e, portanto, a resiliência familiar e construtos rela- 
cionados. Outro fator que pode ter contribuido para esse resultado consiste na amostra apresentar reduzido número de familias participantes e, portanto, há necessidade de estudos futuros contarem com a colaboração de mais pessoas para que seja possivel a identificação de forma mais concisa dos efeitos da intervenção e, caso necessário, que haja aprimoramentos na estrutura do programa de intervenção.

O Teste Wilcoxon detectou diferenças em relação ao estresse e à depressão dos genitores nos diferentes grupos no pré-teste, ou seja, anteriormente à implementação do programa de intervenção. Com base na média dessas variáveis, foi possivel identificar niveis mais elevados desses problemas psicológicos em pais e mães pertencentes ao grupo experimental do que os que compunham o grupo controle, o que pode ter representado um viés nos resultados acerca da avaliação de impacto dos efeitos da intervenção. Nesse sentido, talvez distintos efeitos pudessem ter sido encontrados nas famílias em GE, caso os membros não apresentassem estresse e depressão ou os apresentassem em niveis mais baixos do que os encontrados. A randomização dos participantes não é exigida para estudos com delineamento quase experimental (Santos, 2007). Todavia, destaca-se que ainda que fosse necessária, é algo difícil de se conseguir quando se elabora um projeto com seres humanos, já que a randomização sempre irá ocorrer entre aqueles que aceitarem participar da pesquisa, e não entre todos aqueles que possuam determinadas características e que o pesquisador gostaria que participassem (Baptista \& Morais, 2007). Acredita-se, assim, que o pareamento dessas variáveis entre os participantes dos grupos pode ser considerado uma estratégia eficaz para controlar esse suposto viés. Portanto, mais estudos são recomendados. Cabe destacar que, à época do pré-teste, a utilização do ISSL era considerada favorável pelo Conselho Federal de Psicologia para avaliar o estresse. Embora o Conselho, a partir do pós-teste tenha passado a classificar esse instrumento como desfavorável ao uso em função de seus estudos normativos estarem vencidos, optou-se pela sua permanência na coleta de dados, já que: (a) não foi encontrado teste favorável, que se propõe a medir tal problema psicológico no sistema Conselhos, (b) a modificação do instrumento ao longo da realização da pesquisa poderia trazer prejuizos na análise da variabilidade do estresse, comprometendo a concisão dos resultados, uma vez que o mesmo seria avaliado por meio de instrumentos diferentes.

$\mathrm{Na}$ avaliação de processo dos efeitos do trabatho, percebe-se indicadores positivos em relação ao serviço implementado. Assim, as famílias reconhecem, principalmente, a melhora na comunicação familiar e conjugal, além da aquisição de conhecimentos acerca da SD como consequências de terem participado da intervenção. A comunicação clara, aberta e honesta tende a representar um recurso eficaz para resolução de conflitos, o que contribuiu para o bom funcionamento familiar, levando ao desenvolvimento de processos de resiliência familiar (Caples et al., 2018). Nesse sentido, Choi e Yoo (2015) apontaram que, em seu estudo, os genitores de crianças com SD que apresentavam boa capacidade de comunicação estavam melhor adaptados às adversidades do que aqueles que não a tinham. Acredita-se, portanto, que modificar a comunicação do grupo familiar represente o primeiro passo para que os outros processos-chave possam ser acessados e trabalhados de forma que favoreçam indicativos de resiliência familiar. Assim, esses resultados são considerados otimistas pelas pesquisadoras.

No que tange ao conhecimento relacionado à sindrome, considera-se que quanto maior seja este, mais condição a familia tem de reconhecer as limitações, bem como as habilidades da criança com SD e assim, que essa não seja estigmatizada ou identificada como anormal ou defeituosa. $O$ acesso à informação favorece, portanto, que a criança seja vista como capaz de alcançar metas de seu desenvolvimento, o que resulta na promoção de sua maior autoestima e de sua capacidade de participação na vida familiar (Pereira-Silva \& Almeida, 2014). Além disso, conhecer a sindrome e 
suas caracteristicas tende a minimizar a ocorrência de negação do diagnóstico e a ênfase em sentimentos negativos como, culpa, ressentimento, vergonha, dentre outros (Choi et al., 2011; Koros \& Harrahs, 2017; Rooke et al., 2017), o que favorece o enfrentamento saudável das adversidades relacionadas à sindrome e, portanto, processos de resiliência familiar.

Durante a intervenção no grupo experimental, o preenchimento do diário de campo possibilitou que se percebesse que não houve ocorrência de imprevistos durante as oficinas nem momentos de dificuldades. Além disso, notou-se que as oficinas, principalmente acerca do conhecimento da $\mathrm{SD}$, foram mais benéficas para algumas familias. Optou-se pelas oficinas terem o mesmo conteúdo para todas as familias, todavia, acredita-se que em momentos como esses talvez fosse melhor explorar as informações já existentes para que a oficina fosse adaptada de forma que cada família pudesse aprimorar seus conhecimentos de forma mais equitativa.

Vale ressaltar que se acredita que as familias que aceitaram colaborar com esse estudo representem aquelas que estão implicadas no que tange à promoção do desenvolvimento da criança com SD, o que tende a influenciar à resiliência familiar. Portanto, há de se considerar que, possivelmente, existe um viés de seleção da amostra que pode ter influenciado a validade interna do estudo. Nesse sentido, as familias recrutadas tendem a estar motivadas para participar da intervenção, o que pode inflar os resultados positivos e favoráveis ao trabalho. A partir da realização de mais estudos acerca dessa temática, preferencialmente com maior amostra de participantes e durabilidade da coleta de dados, acredita-se que essas limitações possam ser minimizadas e, assim, possa-se analisar de forma mais concisa a resiliência familiar.

Para garantir resultados mais válidos o ideal teria sido que, além de um grupo controle inativo, existisse também um grupo controle ativo ou inócuo (APA, 2002). Ademais, houve dificuldade na escolha de um tema que pudesse não se relacionar à resiliência familiar. Além disso, as famílias de crianças com SD tendem a ter muitas tarefas a serem realizadas, levando em conta que além das demandas típicas do dia a dia, existem também aquelas relacionadas às especificidades do cuidar de um filho com deficiência, por exemplo, levar aos serviços de tratamento (Pereira-Silva \& Almeida, 2014). Nesse sentido, acredita-se que dependendo do assunto da intervenção no grupo controle ativo, essas famílias não demonstrariam interesse em despender seu tempo para participar, ocasionando a não realização do grupo.

A utilização de critérios específicos para a avaliação dos programas de intervenção, como encontrado em APA (2002), tem sido realizada recentemente na área de Psicologia (Card \& Barnett, 2015: Durgante \& Dell'Aglio, 2018). Para Durgante e Dell'Aglio (2018), tais critérios podem ser considerados pré-requisitos mínimos ou desejáveis, que devem ser satisfeitos/atingidos, para que se possa considerar que o programa de intervenção foi submetido a testes, cumprindo com rigor científico, e apresenta resultados concisos. Assim, faz-se necessário que os pesquisadores utilizem as diretrizes, como em APA (2002), porém avaliando a aplicabilidade dessas de acordo com as especificidades da intervenção e do contexto.

\section{Referências}

Abbasi, S., Sajedi, F., Hemmati, S., Najafi Fard, T., Azadchehr, M. J., \& Poursadoghi, A. (2016). Evaluation of quality of life in mothers of children with Down syndrome. Journal of Practice in Clinical Psychology, 4(2), 81-88. http://dx.doi.org/10.15412/J.JPCP.06040202

Aburawi, E. H., Nagelkerke, N., Deeb, A., Abdulla, S., \& Abdulrazzaq, Y. M. (2015). National growth charts for United Arab Emirates children with Down syndrome from birth to 15 years of age. Journal of Epidemiology, 25(1), 20-29. https://doi.org/10.2188/jea.JE20130081

Alexander, T., \& Walendzik, J. (2016). Raising a child with Down syndrome: Do preferred coping strategies explain differences in parental health? Psychology, 7. 28-39. http://dx.doi.org/10.4236/psych.2016.71005

American Psychological Association, APA (2002). Criteria for evaluating treatment guidelines. American Psychologist, 57(12), 1052-1059. http://dx.doi.org/10.1037/ $0003-066 \times .57 .12 .1052$

Azevedo, A. P. S., \& Damke, A. S. (2017). A criança com sindrome de Down: O sentido da inclusão no contexto da exclusão. Revista Educação Especial, 30(57), 103-114. http://dx.doi.org/10.5902/1984686X17862 
Baptista, M. N., \& Morais, P. R. (2007). Delineamento experimental. In M. N. Baptista, \& D. C. Campos (Orgs.), Metodologias de pesquisa em ciências: Análises quantitativa e qualitativa (pp.122-138). LTC.

Bardin, L. (2011). Análise de conteúdo. Lisboa: Edições 70.

Beck, A. T., Steer, R. A., \& Brown, G. H. (1996). BDI-II: Beck depression inventory manual. Psychological Corporation.

Caples, M., Martin, A-M., Dalton, C., Marsh, L., Savage, E., Knafl, G., \& Van Riper, M. (2018). Adaptation and resilience in families of individuals with Down syndrome living in Ireland. British Journal of Learning Disabilities, 1-9. https://doi.org/10.1111/bld.12231

Card, N. A., \& Barnett, M. A. (2015). Methodological considerations in studying individual and family resilience. Family Relations, 64, 120-133. https://doi.org/10.1111/ fare.12102

Choi, E. K., Lee, Y. J., \& Yoo, I. Y. (2011). Factors associated with emotional response of parents at the time of diagnosis of Down syndrome. Journal for Specialists in Pediatric Nursing, 16, 113-120. https://doi.org/10.1111/j. 1744-6155.2011.00276.X

Choi, H., \& Van Riper, M. (2016). Maternal perceptions to open-ended questions about life with Down syndrome in Korea. The Qualitative Report, 21(2), 288-298. https:// nsuworks.nova.edu/tqr/vol21/iss2/7

Choi, E. K., \& Yoo, I. Y. (2015). Resilience in families of children with Down syndrome in Korea. International Journal of Nursing Practice, 21, 532-541. https://doi. org/10.1111/ijn.12321

Crnic, K. A., Neece, C. L., McIntyre, L. L., Blacher, J., \& Baker, B. L. (2017). Intellectual disability and developmental risk: Promoting intervention to improve child and family well-being. Child Development, 1-10. https:// doi.org/10.1111/cdev.12740

Dempsey, I., \& Keen, D. (2008). A review of processes and outcomes in family-centered services for children with a disability. Topics in Early Childhood Special Education, 28, 42-52. https://doi.org/10.1177/0271121408316699

Dessen, M. A., \& Braz, M. P. (2005). A familia e suas inter-relações com o desenvolvimento humano. In M. A. Dessen, \& A. L. Costa Jr. (Orgs.), A ciência do desenvolvimento humano: tendências atuais e perspectivas futuras (pp. 113-131). Artmed.

Dessen, M. A., \& Cerqueira-Silva, S. (2009). Desenvolvendo sistemas de categorias com dados de entrevistas. In L. Weber \& M. A. Dessen (Eds), Pesquisando a Familia: Instrumentos para coleta e análise de dados (pp. 43-56) Curitiba: Juruá

Durgante, H., \& Dell'Aglio, D. D. (2018). Critérios metodológicos para a avaliação de programas de intervenção em psicologia. Avaliação Psicológica, 17(1), 155-162. http://dx.doi.org/10.15689/ap.2017.1701.15.13986

Field, A. (2009). Descobrindo a estatistica usando o SPSS (L. Viali, Trad.). Artmed.
Gerstein, E. D., Crnic, K. A., Blacher, J., \& Baker, B. L. (2009). Resilience and the course of daily parenting stress in families of young children with intellectual disabilities. Journal of Intellectual Disability Research, 53(12), 981-997.

Goff, B. S. N., Monk, J. K., Malone, J., Staats, N., Tanner, A., \& Springer, N. P. (2016). Comparing parents of children with Down syndrome at different life span stages. Journal of Marriage and Family, 78(4), 1131-1148. https:// doi.org/10.1111/jomf.12312

Gorenstein, C., Pang, W. Y., Argimon, I. L., \& Werlang B. S. G. (2011). Manual do inventário de depressão de Beck - BDI-II. Casa do Psicólogo.

Grant, S., Cross, E., Wraith, J. E., Jones, S., Mahon, L., Lomax, M., ... Hare, D. (2013). Parental social support, coping strategies, resilience factors, stress, anxiety and depression levels in parents of children with MPS III (Sanfilippo syndrome) or children with intellectual disabilities (ID). Journal of Inherited Metabolic Disease, 36, 281-291.

Koros, E. J., \& Harrahs, M. (2017). Coping with Down's Syndrome: A Case Study of Parents with Children Suffering from Down's Syndrome in Kenya. Journal of Special Needs and Disabilities Studies, 1(3), 1-21.

Lipp, M. E. N. (2000). Inventário de Sintomas de Estresse para Adultos. Casa do Psicólogo.

Marchal, J. P., van Oers, H. A., Maurice-Stam, H., Grootenhuis, M. A., van Trotsenburg, A. S. P., \& Haverman, L. (2017). Distress and everyday problems in Dutch mothers and fathers of young adolescents with Down syndrome. Research in Developmental Disabilities, 67. 19-27. https://doi.org/10.1016/j.ridd.2017.05.005

Mazzeo, V. (2007). Los cambios en la organización familiar: El incremento de las familias monoparentales en la Ciudad de Buenos Aires a partir de los ochenta. Población de Buenos Aires, 4(5), 63-74.

Nussbaum, R. L., McInnes, R. R., \& Willard, H. F. (2015). Thompson and Thompson genetics in medicine. Elsevier Health Sciences.

Oñate, L., \& Calvete, E. (2017). Una aproximación cualitativa a los factores de resiliencia en familiares de personas con discapacidad intelectual en España. Psychosocial Intervention, 26(2), 93-101. https://doi. org/10.1016/j.psi.2016.11.002

Padesky, C. A., \& Mooney, K. A. (2012). Strengths-based cognitive-behavioural therapy: A four-step model to build resilience. Clinical Psychology and Psychotherapy, 19, 283-290. https://doi.org/10.1002/cpp.1795

Pereira-Silva, N. L., \& Almeida, B. R. (2014). Reações, sentimentos e expectativas de familias de pessoas com necessidades educacionais especiais. Psicologia Argumento, 32(79), 111-122. http://dx.doi.org/10.7213/ psicol..argum.32.502. $\mathrm{AO} 10$

Raposo, H. S., Figueiredo, B. F. C., Lamela, D. J. P. V., Nunes-Costa, R. A., Castro, M. C., \& Prego, J. (2009). Ajustamento da criança à separação ou divórcio dos pais. Revista de Psiquiatria Clínica, 38(1), 29-33. http:// dx.doi.org/10.1590/S0101-60832011000100007 
Roizen, N. J., Magyar, C. I., Kuschner, E. S., Sulkes, S. B., Druschel, C., van Wijngaarden, E., Rodgers, L., Diehl, A., Lowry, R. \& Hyman, S. L. (2014). A community cross-sectional survey of medical problems in 440 children with Down syndrome in New York state. Journal of Pediatrics, 164, 871-875. https://doi.org/10.1016/j.jpeds.2013.11.032

Rooke, M. I. (2014). Familias com filho com sindrome de Down: Investigando a resiliência familiar [Dissertação de mestrado, Universidade Federal de Juiz de Fora]. http:// repositorio.ufjf.br:8080/jspui/bitstream/ufjf/863/1/ mayseitagibarooke.pdf

Rooke, M. I. (2019). Resiliência em famílias de crianças com sindrome de Down: Desenvolvimento, implementação de uma intervenção e avaliação de seus efeitos [Tese de doutorado, Universidade Federal de Juiz de Fora, Juiz de Fora]. https://repositorio.ufjf.br/jspui/ bitstream/ufff/9980/2/mayseitagibarooke.pdf

Rooke, M. I., Almeida, B. R., \& Mejía, C. F. (2017). Intervenção com familias de pessoas com deficiência intelectual: Análise da produção científica. Revista de Psicologia, 8(2), 92-100

Rooke, M. I., \& Pereira-Silva, N. L. (2016). Indicativos de resiliência familiar em famílias de crianças com sindrome de Down. Estudos de Psicologia, 33, 117-126. http://dx.doi.org/10.1590/1982-027520160001000012

Sánchez-Teruel, D., \& Robles-Bello, M. A. (2015). Respuesta a un programa de resiliencia aplicado a padres de niños con Sindrome de Down. Universitas Psychologica, 14(2), 645-658. https://doi.org/10.11144/Javeriana. upsy14-1.rpra

Santos, A. A. A. (2007). Delineamento quase-experimental. In M. N. Baptista \& D. C. Campos (Orgs.), Metodologias de pesquisa em ciências: Análises quantitativa e qualitativa (pp.139-146). LTC.

Scorgie, K., \& Sobsey, D. (2000). Transformational outcomes associated with parenting children who have disabilities. Mental Retardation, 38(3), 195-206. https:// doi.org/10.1352/0047-6765(2000)038<0195:TOAWPC>2.0. $\mathrm{CO}: 2$

Van Riper, M. (2007). Families of children with Down syndrome: Responding to "a change in plans" with resilience. Journal of Pediatric Nursing, 22, 116-128. https://doi.org/10.1016/j.pedn.2006.07.004

Walsh, F. (2016). Applying a family resilience framework in training, practice, and research: Mastering the art of the possible. Family Process, 55, 616-632. https://doi. org/10.1111/famp.12260

\section{Mayse Itagiba Rooke}

Doutora e mestre em Psicologia pela Universidade Federal de Juiz de Fora (UFJF), em Juiz de Fora, MG, Brasil. Professora da Universidade Anhembi Morumbi (UAM), em São José dos Campos, SP, Brasil.

\section{Nara Liana Pereira Silva}

Doutora e mestre em Psicologia pela Universidade de Brasilia (UNB), em Brasília, DF, Brasil. Professora da Universidade Federal de Juiz de Fora (UFJF), em Juiz de Fora, MG, Brasil.

\section{Endereço para correspondência}

\section{Mayse Itagiba Rooke}

Universidade Federal de Juiz de Fora

Departamento de Psicologia - Instituto de Ciências Humanas

Campus Universitário

Rua José Lourenço Kelmer

36036-900

Juiz de Fora, MG, Brasil

Os textos deste artigo foram revisados pela Poá Comunicação e submetidos para validação das autoras antes da publicação. 